\title{
Trilhas de Aprendizagem em Ambientes Virtuais de Ensino- aprendizagem: Uma Revisão Sistemática da Literatura
}

\author{
David Brito Ramos ${ }^{1,3}$, Elaine Harada Teixeira de Oliveira ${ }^{1}$, Ilmara M. M. \\ Ramos $^{1,3}$, Kelson M. T. Oliveira ${ }^{2}$ \\ ${ }^{1}$ Instituto de Computação - Universidade Federal do Amazonas (UFAM) - Manaus - \\ AM - Brasil \\ ${ }^{2}$ Instituto de Ciências Exatas - Universidade Federal do Amazonas (UFAM) - Manaus \\ - AM - Brasil \\ ${ }^{3}$ Instituto Federal de Educação, Ciência e Tecnologia do Amazonas (IFAM) - Campus \\ Parintins - Parintins - AM - Brasil \\ davidness@gmail.com, elaine@icomp.ufam.edu.br, \\ ilmaramonteverde@gmail.com, kelsonmota@ufam.edu.br
}

\begin{abstract}
The research presented in this paper is the result of a Systematic Literature Review that aimed to identify how learning paths are represented within the e-learning systems. It was also verified if these representations include not only data structure and modeling of e-learning systems, but also the visualization of learning paths by teachers and students.

Resumo. A pesquisa apresentada neste trabalho é resultado de uma Revisão Sistemática da Literatura que buscou identificar como as trilhas de aprendizagem são representadas nos sistemas e-learning. Foi verificado se essas representações contemplam apenas a estrutura de dados e modelagem dos sistemas de e-learning, ou se também há visualização das trilhas de aprendizagem pelos professores e alunos.
\end{abstract}

\section{Introdução}

O uso da Internet como facilitador da comunicação tem se mostrado útil em diversas áreas de conhecimento. Com o melhoramento das conexões e a ampliação do acesso à Internet, muitas pesquisas têm voltado seus esforços para o melhoramento dos ambientes virtuais de aprendizagem. Para que estas mudanças de melhoria aconteçam, está claro que é necessário conhecer o aluno. Nesse sentido muitas técnicas estão sendo aplicadas, entretanto para a maioria delas é necessária a solicitação explícita de dados do aluno, tendo como fonte direta de informações unicamente o próprio aluno, como nos trabalhos de Ferreira, Barbosa e Gluz (2013), de Müller e Silveira (2013) e Gomes e Pimentel (2013). Assim os dados coletados podem não corresponder à situação real do aluno. Para evitar esse problema, existem trabalhos que buscam monitorar, de forma transparente, as atividades dos alunos no intuito de encontrar padrões de comportamento que possam elucidar possíveis problemas ou melhoras no aprendizado. Uma das formas de monitoramento do aluno pode ser realizada através da observação das ações que ele executa dentro do sistema, sendo que estas formam caminhos que são conhecidos como trilhas de aprendizagem. Nesse sentido, esta Revisão Sistemática busca identificar como essas trilhas são representadas, uma vez que elas descrevem de forma natural o comportamento do aluno. 
A seguir, a seção 2 define o contexto da pesquisa. A seção 3 aborda o método. A seção 4 apresenta o processo de condução da revisão. Na seção 5 são realizadas a análise e discussão dos dados. Na seção 6 são apresentadas as limitações da revisão e por fim são feitas as considerações finais.

\section{Contexto da Pesquisa}

\subsection{Ambientes Virtuais de Aprendizagem}

Um Ambiente Virtual de Aprendizagem ou Ensino-Aprendizagem (AVA), também conhecido como LMS (Learning Management System) ou VLE (Virtual Learning Environment), de acordo com Ninoriya, Chawan e Meshram (2011), é uma ferramenta que permite o gerenciamento de cursos online, registro de alunos e a avaliação de seus resultados de aprendizagem. Segundo Silva (2011), um AVA busca permitir a construção do conhecimento por meio da interatividade e interação entre pessoas ou grupos e não apenas disponibilizar conteúdo.

\subsection{Trilhas de Aprendizagem}

De acordo com Tafner, Tomelin e Müller (2012), as trilhas de aprendizagem são caminhos virtuais para o desenvolvimento intelectual que promovem e desenvolvem competências.

No contexto de desenvolvimento de ferramentas educacionais, nos trabalhos da literatura relacionados aos AVAs, as trilhas de aprendizagens, são consideradas sob dois pontos de vista: a trilha é a sequência de conteúdo e atividades definida pelo professor, ao planejar sua disciplina e, posteriormente, disponibilizá-la no ambiente virtual; ou a trilha é aquela percorrida pelo aluno, durante a sua interação com recursos disponibilizados no ambiente virtual. Trilhas de aprendizagem também são referenciadas como: caminhos de aprendizagem, percurso de aprendizagem e em língua inglesa: learning path, learning route, learning itineraries.

\section{Metodologia}

Normalmente, as pesquisas iniciam com uma revisão da literatura, uma vez que o estudo de trabalhos relacionados ajuda no processo de criação de um novo conhecimento. O resultado de uma revisão abrangente é, indubitavelmente, fator determinante no desenvolvimento da pesquisa. Para Kitchenham e Charters (2007) a pesquisa deve ser minuciosa e para ter valor científico, sendo esta a principal razão para a realização de Revisões Sistemáticas da Literatura (RSL).

Trabalhos recentes dentro do escopo de ensino-aprendizagem têm sido publicados como RSL. Pontes et al (2014), apresenta uma RSL sobre filtragens de recomendação de Objetos de Aprendizagens publicados no SBIE. O trabalho de ZemLopes et al (2013) busca identificar como tecnologias da Web Semântica são utilizadas em ambientes educacionais e quais as principais técnicas empregadas. O trabalho de Fassbinder, Delamaro e Barbosa (2014), apresenta uma RSL sobre Massive Open Online Courses (MOOCs) que busca definir, categorizar e caracterizar o ambiente dos MOOCs.

Este trabalho está baseado em um estudo aprofundado sobre trilhas de aprendizagem, realizado por meio de uma RSL, seguindo as orientações de Kitchenham 
(2004) (o resultado completo está registrado em Ramos e Oliveira (2015)). A revisão realizada pode ser classificada como uma revisão quasi-sistemática, uma vez que não houve comparações entre publicações, pois buscou-se realizar uma pesquisa de cunho exploratório. Durante o processo da RSL utilizou-se uma ferramenta especialmente para este fim, chamada de StArt, desenvolvida na Universidade de Federal de São Carlos, pelo Laboratório de Pesquisa em Engenharia de Software (LaPES) e está disponível no endereço lapes.dc.ufscar.br. A versão 2.0 foi utilizada para auxiliar a pesquisa.

\subsection{Objetivo e questão de pesquisa}

O objetivo da RSL foi identificar como as trilhas de aprendizagem são representadas dentro dos sistemas e-learning e se existe uma representação visual dessas trilhas para os professores e alunos. A descrição do objetivo conforme o paradigma Goal-QuestionMetric de Basili, Caldiera e Rombach (1984), pode ser observada abaixo:

Analisar publicações científicas através de um estudo baseado em revisão sistemática, com o propósito de identificar como são representadas as trilhas de aprendizagem em Ambientes Virtuais de Aprendizagem com relação à representação dessas trilhas para os sistemas, professores e alunos do ponto de vista dos pesquisadores no contexto acadêmico ou industrial.

A RSL buscou responder à questão principal: como as trilhas de aprendizagem são representadas dentro dos sistemas de e-learning?

\subsection{Método de busca das publicações}

Utilizou-se as máquinas de buscas específicas, das bibliotecas selecionadas, sendo consultados os conjuntos de palavras-chave pré-estabelecidos na pesquisa.

Apenas fontes digitais foram consideradas para consulta, sendo estas: IEEE Computer Science Digital Library <http://ieeexplore.iee.org> e Scopus $<$ http://www.scopus.com>. A Scopus destaca-se por armazenar publicações de diversas fontes como, por exemplo: Springer, ACM, ScienceDirect/Elsevier, British Computer Society.

\subsection{Expressão de Busca}

A expressão de busca foi definida após a execução de testes, auxiliados com base em artigos de controle, composta por palavras-chave que representam a população $(\mathrm{P})$ e a intervenção (I) (maiores detalhes estão descritos em Ramos e Oliveira (2015)). A composição básica da expressão de busca possui a seguinte estrutura: (P) AND (I). Para a busca na Scopus, as aspas (“") foram substituídas por chaves $(\{\})$.

A população é descrita por um conjunto de palavras relacionadas a Learning Management Systems e e-Learning, enquanto a intervenção descreve palavras relacionadas a Learning Path. A Tabela 1, a seguir, apresenta as palavras-chave da expressão de busca.

\section{Tabela 1 - Termos de busca}

\section{População}

"learning management system", "learning management systems", "lms", "virtual learning environment", "virtual learning environments", "vle", "course management system", "course management systems", "intelligent tutoring system", "intelligent tutoring systems", "coaching 
assisted system", "coaching assisted systems", "moodle", "distance course", "distance education", "distance learning", "distance training course", "online learning", "distance courses", "distance training courses", "e-learning framework", "e-learning system", "e-learning systems", "e-learning module", "e-learning modules", "e-learning recommender system", "elearning recommender systems", "e-learning program", "e-learning programs", "e-learning adaptive system", "e-learning adaptive systems", "e-learning path", "e-learning paths", "elearning setting", "e-learning settings", "distant learning system" e "distant learning systems"

\section{Intervenção}

"learning path", "learning itinerary", "learning route", "learning workflow", "individual path", "learning paths", "learning itineraries", "learning routes", "learning workflows" e "individual paths"

\subsection{Critérios de Inclusão e Exclusão}

Os critérios de inclusão (CI) e de exclusão (CE), listados na Tabela 2, foram elaborados a partir dos artigos de controle.

\section{Tabela 2 - Critérios de Inclusão e Exclusão}

\section{Critérios de Inclusão}

(CI1) Artigos que apresentem representações de modelos, visuais ou não, sobre trilhas de aprendizagem.

(CI2) Artigos que apresentem propostas de adaptação de trilhas de aprendizagem em ambientes virtuais.

\section{Critérios de Exclusão}

(CE1) Não serão selecionadas publicações em que as palavras-chave da busca não apareçam no título, resumo e/ou palavras-chave.

(CE2) Não serão selecionadas publicações em que as siglas equivalentes não signifiquem: LMS - "Learning Management System, VLE - "Virtual Learning Environment".

(CE3) Serão excluídas publicações que não tem disponibilidade de conteúdo para leitura (por exemplo, onde os trabalhos são pagos ou não disponibilizados pelas máquinas de buscas).

(CE4) Artigos que apenas apresentam relatos de experiência de algum modelo, técnica ou ferramenta.

(CE5) Artigos que apenas citam sistemas de e-learning (LMS, VLE, etc.), mas não o abordam como foco principal.

(CE6) Não serão selecionadas publicações que não relacionam Trilhas de Aprendizagem e Ambientes Virtuais de Aprendizagem (LMS, CMS, ITS, LCMS).

(CE7) Não serão selecionadas publicações que apresentam ambientes virtuais de aprendizagem, mas não descrevam trilhas de aprendizagem.

(CE8) Não serão selecionadas publicações que descrevam trilhas de aprendizagem, mas não relacionados a ambientes virtuais de aprendizagem.

\subsection{Extração de Dados}

Foram extraídos dados básicos da publicação como título, autores, local de publicação, ano de publicação, palavras-chave e também dados relativos às características que auxiliaram na resposta da questão de pesquisa e também na caracterização das publicações. São eles: técnicas utilizadas no trabalho, ambiente virtual utilizado, tipo AVA, público-alvo no trabalho, abrangência do trabalho, tipo da pesquisa, momento de 
criação das trilhas de aprendizagem, como as trilhas são representadas, se a ferramenta da publicação apresentada recomenda trilhas de aprendizagem e quais os nós das trilhas.

\section{Condução de Revisão}

Após o processo de planejamento da revisão, realizou-se a fase de execução. É importante informar que a execução da expressão de busca ocorreu em junho de 2014 . Primeiramente, os estudos preliminares foram identificados através da execução da expressão de busca. Ao todo foram retornados 279 artigos, sendo 201 artigos da base da Scopus e 78 da base da IEEE, os 60 trabalhos duplicados foram identificados posteriormente via software e revisão manual, portanto foram analisadas, no primeiro filtro, 219 publicações.

No primeiro filtro, os trabalhos foram analisados de acordo com o título, resumo e palavras-chave. Publicações cujo conteúdo para leitura não pode ser obtido diretamente da fonte de pesquisa (Scopus e IEEE), foram pesquisadas via máquina de busca (google.com) e/ou quando possível, os autores eram contatados via e-mail ou pelo portal researchgate.net. Esgotando-se as possibilidades, os trabalhos foram classificados conforme critérios estabelecidos na fase de planejamento (Figura 1).

$\mathrm{Na}$ fase do Segundo Filtro, somente os 127 trabalhos aceitos na fase anterior foram lidos na íntegra. Também foram adotados os mesmos critérios de inclusão e exclusão do primeiro filtro. Portanto, ao todo 76 trabalhos foram aceitos nesta última fase e formaram a base para a extração de dados (Figura 2).

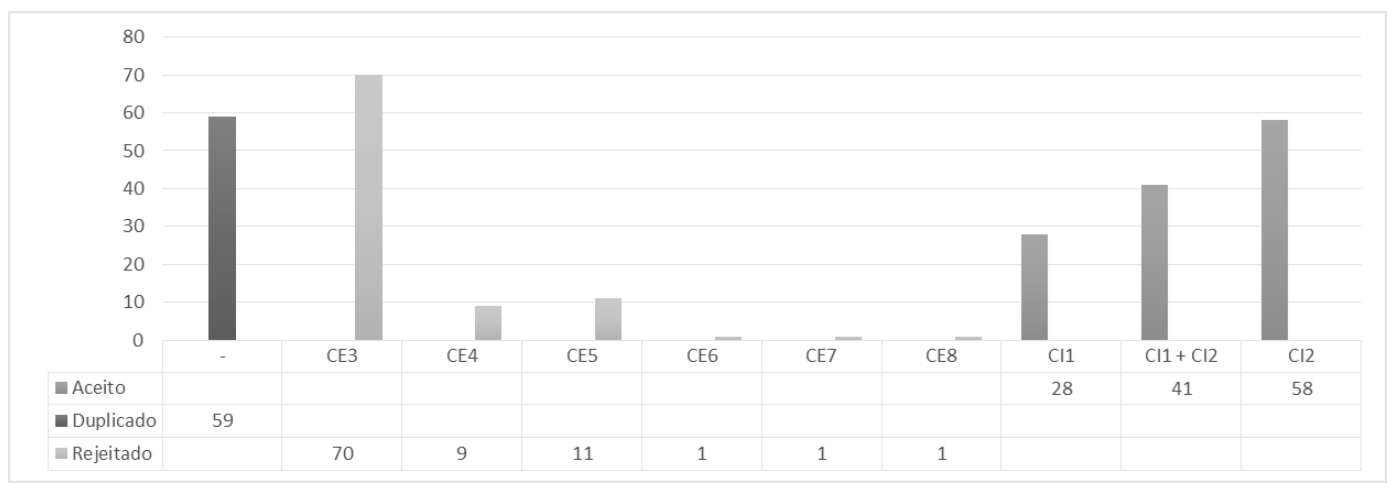

Figura 1 - Resultado do Primeiro Filtro por Critério

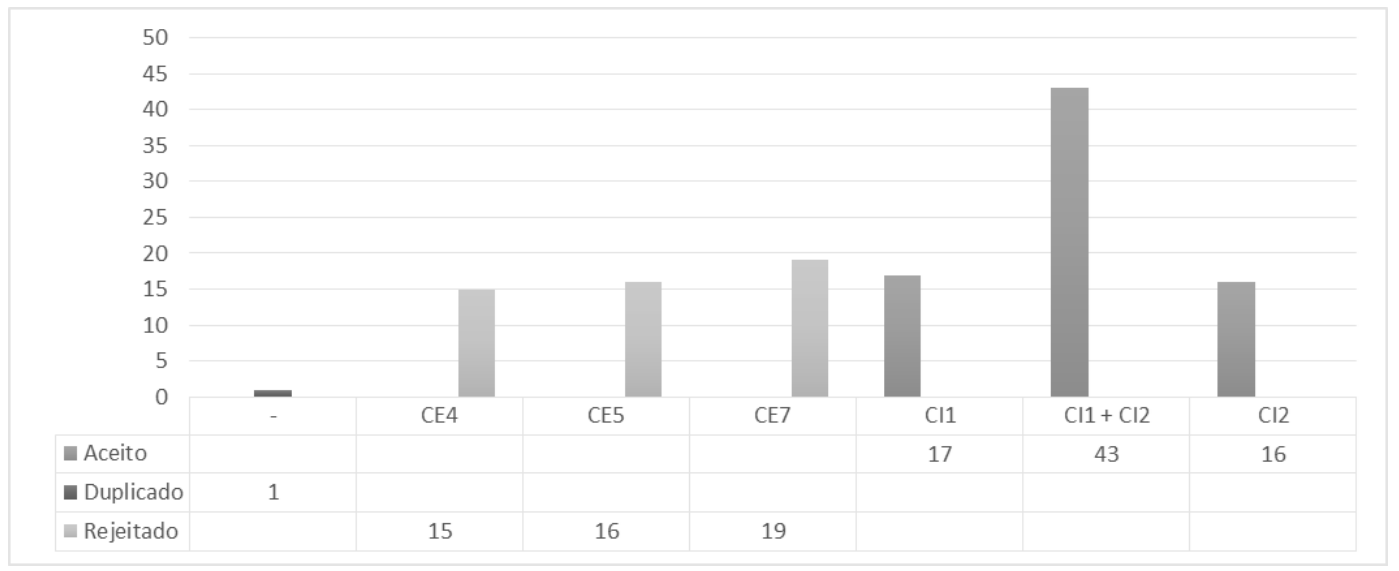

Figura 2 - Resultado do Segundo Filtro por Critério 


\section{Análise e Discussão dos Dados}

\subsection{Resposta à questão principal}

A partir dos dados das publicações aceitas foi possível estabelecer como as trilhas de aprendizagem são representadas nos ambientes virtuais, quais nós as compõem, em que momentos são geradas e se há recomendação de trilhas de aprendizagem.

Os métodos de representação de trilhas de aprendizagem mais utilizados são Grafos $(39,47 \%)$ e Sequenciamento $(30,26 \%)$. Trabalhos que utilizam grafos podem explorar uma flexibilização em tempo real da aprendizagem. O aprendiz pode, ao longo de todo o processo de estudo e em cada parte dele, mudar suas características e isso pode ser avaliado constantemente em seu perfil. Já o sequenciamento implica que, em um determinado intervalo do curso, o aprendiz deverá percorrer uma sequência de atividades que não poderá ser mudada, ou seja, o aluno é avaliado periodicamente, mas somente entre as sequências, de forma que as características como conhecimento adquirido, estilo de aprendizagem, perfil cognitivo e outros dados sobre o aprendiz sejam atualizados e utilizados como parâmetros para a geração da sequência de atividades seguintes. A Tabela 3 apresenta todas as representações de trilhas de aprendizagem encontradas na revisão.

Tabela 3 - Representação das trilhas de aprendizagem

\begin{tabular}{|l|c|l|c|}
\hline Como as trilhas são representadas & Total & Como as trilhas são representadas & Total \\
\hline Grafo & 30 & Cadeia de Cromossomos & 2 \\
\hline Sequenciamento & 23 & Árvore & 1 \\
\hline Rede de Petri & 4 & Vetor & 1 \\
\hline Ontologia & 4 & Mapa 3d_SLN & 1 \\
\hline Fluxo de trabalho (workflow) & 3 & Espaço de Estados de Aprendizagem & 1 \\
\hline Gráfico & 3 & Plano - Case-Based planning (CBP) & 1 \\
\hline Relacionamento Conceito-Efeito & 2 & & \\
\hline
\end{tabular}

Os termos mais citados com relação aos nós das trilhas de aprendizagem são: Objetos de Aprendizagem (OA), Conceitos, Módulos e Atividades. Observa-se que esses nós mais citados possuem uma visão macro do processo de aprendizagem, assim não representam especificamente um recurso, mais sim um agregado de recursos estáticos e/ou dinâmicos representados de forma única. Uma atividade envolve, por exemplo, o contato com os conhecimentos teóricos e a aplicação em um ou mais exercícios. A Tabela 4 apresenta todos os termos citados nas publicações analisadas, com destaque para dois trabalhos que citam a intervenção do professor/tutor como um nó da trilha de aprendizagem. Caso a publicação tenha afirmado trabalhar com OA e descreve alguns destes, por exemplo, como fóruns, vídeos e tarefas então os nós das trilhas de aprendizagem são registrados na extração de dados como dos tipos OA, fóruns, vídeos e tarefas e não apenas como OA. A mesma ideia vale para Conceitos, Módulos e Atividades. Esta forma de registro foi necessária por causa dos diferentes níveis detalhes das publicações que não podem ser desconsiderados.

Quando o aprendiz percorre um caminho dentro do ambiente de aprendizagem, ele executa uma série de ações, como assistir a um vídeo, acessar uma página externa, ler um conteúdo, realizar um teste, postar em um fórum, entre outras que lhe são permitidas. O conjunto de ações possíveis pode ser especificado antes mesmo do 
aprendiz interagir com o AVA, neste, cria-se um caminho padrão e uma série de caminhos alternativos, dessa forma é possível prever a próxima ação que será disponibilizada para o aprendiz. Para sistemas de e-learning com estas características, denominou-se o momento da geração das trilhas de aprendizagem como préestabelecido. Existem 36 publicações, conforme Tabela 5, com esta característica. As demais publicações foram classificadas como de tempo real, isto significa que, apesar do ambiente virtual possuir em sua base de dados os recursos e atividades que serão disponibilizados aos aprendizes, não é determinado, previamente, qual atividade ou recurso será fornecido após a conclusão da ação anterior.

Tabela 4 - Nós das trilhas de aprendizagem

\begin{tabular}{|l|c|l|c|l|c|}
\hline \multicolumn{1}{|c|}{ Tipos de nó } & Total & \multicolumn{1}{|c|}{ Tipos de nó } & Total & \multicolumn{1}{c|}{ Tipos de nó } & Total \\
\hline $\begin{array}{l}\text { Objetos de } \\
\text { Aprendizagem }\end{array}$ & 31 & $\begin{array}{l}\text { Material Web/Conteúdo } \\
\text { Externo }\end{array}$ & 4 & Laboratório virtual & 2 \\
\hline Conceitos & 22 & Página Web & 4 & Seminário & 1 \\
\hline Atividades & 16 & Comentário das questões & 3 & Falar em publico & 1 \\
\hline Módulos & 16 & Fórum & 2 & Curso alto nível & 1 \\
\hline Questionários & 12 & Chat & 2 & Animações 3D & 1 \\
\hline Tarefas & 11 & $\begin{array}{l}\text { Intervenção } \\
\text { Professor/Tutor }\end{array}$ & 2 & Biblioteca virtual & 1 \\
\hline Objetos Multimídia & 9 & Notas Suplementares & 2 & Workshop virtual & 1 \\
\hline Assistir um vídeo & 4 & Livros Recomendados & 2 & Colaboração virtual & 1 \\
\hline & & Resumo & 2 & & \\
\hline
\end{tabular}

Tabela 5 - Geração das Trilhas de Aprendizagem

\begin{tabular}{|l|c|}
\hline Momento de criação das trilhas de aprendizagem & Total \\
\hline Tempo real & 40 \\
\hline Pré-estabelecido & 36 \\
\hline
\end{tabular}

\subsection{Publicações}

Além do levantamento sobre as questões diretamente relacionadas às trilhas de aprendizagem, também foi possível identificar informações referentes às publicações. A Figura 3, apresenta o número de publicações por ano, considerando apenas os artigos que foram aceitos dentro dos critérios pré-estabelecidos, o que revela um aumento do interesse dos pesquisadores nos últimos dez anos e, consequentemente, a crescente relevância do tema.

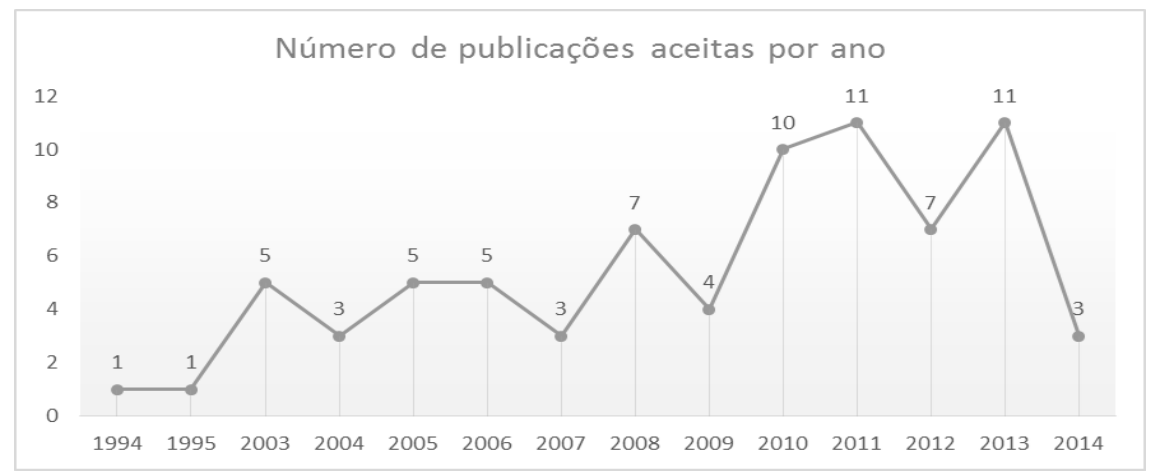

Figura 3- Número de publicações por ano 
Outro destaque é que as publicações estão distribuídas por várias fontes cujos temas não são exatamente sobre Educação, como, por exemplo, Engenharia Elétrica ou Inteligência Artificial. A Tabela 6, apresenta uma visão das principais fontes por contagem de publicações considerando apenas os artigos aceitos.

Tabela 6 - Principais locais de publicação

\begin{tabular}{lc}
\hline \multicolumn{1}{c}{ Local de Publicação } & Total \\
\hline Advanced Learning Technologies (ICALT), IEEE International Conference on & 9 \\
\hline Computers and Education & 5 \\
\hline Expert Systems with Applications & 4 \\
\hline $\begin{array}{l}\text { Lecture Notes in Computer Science (including subseries Lecture Notes in Artificial } \\
\text { Intelligence and Lecture Notes in Bioinformatics) }\end{array}$ & 4 \\
\hline Education, IEEE Transactions on & 2 \\
\hline Electronics, Circuits and Systems (ICECS), IEEE International Conference on & 2 \\
\hline Multimedia Computing and Systems (ICMCS), International Conference on & 2 \\
\hline Neural Networks (IJCNN), IEEE International Joint Conference on. (IEEE World & 2 \\
\hline
\end{tabular}

Também verificou-se a abordagem de pesquisa das publicações aceitas para a revisão. Conforme é apresentado na Tabela 7, trinta e seis publicações foram classificadas como pesquisa de validação, ou seja, as técnicas não foram aplicadas na prática, os experimentos desses trabalhos são realizados em laboratório. Vinte e um são pesquisas de avaliação, nesse caso o trabalho possui uma ferramenta que foi aplicada na prática onde as consequências foram avaliadas. Por fim, dezenove publicações são propostas de solução onde foram defendidas a aplicabilidade e possíveis benefícios, mas o trabalho não possui uma ferramenta pronta ou ela está em fase inicial de desenvolvimento e consequente não possui resultados de experimentos.

Tabela 7 - Abordagem da Pesquisa

\begin{tabular}{ll}
\hline Abordagem da Pesquisa & Total \\
\hline Validação & 36 \\
\hline Avaliação & 21 \\
\hline Proposta de solução & 19 \\
\hline
\end{tabular}

\subsection{Conclusões da análise}

Grafo é o método mais utilizado para representar trilhas de aprendizagem (39,47\%). Os termos mais citados para os nós de uma TA são: Objetos de Aprendizagem, Conceitos, Módulos e Atividades. Em geral, as TAs são geradas em tempo real. O Moodle é citado por $13 \%$ dos trabalhos como AVA utilizado. O grande foco de pesquisa nos trabalhos é a adaptação do ambiente virtual de aprendizagem à individualidade do aluno. Verificouse também que $55,26 \%$ dos trabalhos analisados foram publicados somente nos últimos cinco anos, sendo que o trabalho mais antigo foi publicado em 1994. Dentre todos os artigos aceitos para estudo, somente quatro apresentaram as trilhas de forma visual ao usuário (aprendiz, docente), conforme Tabela 8.

\section{Tabela 8 - Publicações que exibem as trilhas visualmente aos aprendizes e docentes}

\begin{tabular}{lc}
\hline \multicolumn{1}{c}{ Artigos que apresentam as trilhas visualmente aos usuários } & Ano \\
\hline How to See Training Paths in Learning Management Systems? & 2010 \\
\hline eGraph tool: graphing the learning process in LMSs & 2014 \\
\hline
\end{tabular}


A Business Process Management Based Virtual Learning Environment : Customised

Learning Paths

Learning maps usage of cartographic metaphors for orientation in e-learning courses

\section{Limitações da Revisão}

A RSL é realizada por meio de protocolo robusto e objetivo que rege todo o processo de revisão, entretanto isso não garante a eliminação por completo da subjetividade inerente aos pesquisadores. Concomitantemente, a exclusão de algum trabalho relevante, eliminado por não corresponder a alguns dos critérios de seleção, é sempre uma possibilidade premente. Nesse sentido, é possível que a execução do primeiro filtro possa ter excluído alguns trabalhos relevantes. Isso porque as publicações que não continham os termos de busca nas palavras-chave, títulos ou resumos são descartadas nessa fase e nenhuma leitura completa é realizada. Outra limitação decorre de decisões subjetivas ocorridas durante o processo de leitura e compreensão dos trabalhos, pois alguns estudos não apresentaram uma descrição clara, dificultando a aplicação direta dos critérios de inclusão e exclusão.

\section{Considerações Finais}

Os estudos mostraram uma grande heterogeneidade para a representação das trilhas de aprendizagem, com destaque para o nível de detalhes de trabalhos que citavam especificamente páginas Web, fórum e chats, por exemplo, ou genericamente, como conceitos/módulos/curso e outros trabalhos que descreviam vários níveis de representação. A maioria dos trabalhos trata as trilhas de aprendizagem como resultado de sua criação e não do processo natural do aluno de percorrer o conteúdo do $e$ learning, mas também há trabalhos que consideram a ótica do aluno. Esta RSL também revela que o grande foco de pesquisa nos trabalhos estudados é a adaptação do ambiente virtual de aprendizagem à individualidade do aluno, seja ela caracterizada por seu perfil cognitivo, estilo de aprendizagem, habilidade ou nível de conhecimento. Há também um crescente aumento de pesquisas que utilizam técnicas de Inteligência Artificial, tais como: agentes, otimização de colônia de formiga, algoritmos genéticos, redes neurais. Muito sistemas propostos são anunciados como sistema de recomendação, porém os conteúdos selecionados são impostos ao aluno.

De posse das informações obtidas, está sendo desenvolvido um modelo de identificação e representação de trilhas de aprendizagem que apresenta uma visualização de ações realizadas no ambiente virtual Moodle.

\section{Agradecimentos}

Os autores agradecem o apoio financeiro da Fundação de Amparo à Pesquisa do Estado do Amazonas (FAPEAM) através do Edital N 016/2013, Projeto PROTI-Pesquisa sob o processo $\mathrm{N}^{\circ} 062.00597 / 2014$ e bolsa de pesquisa. Além disso, parte dos resultados apresentados neste trabalho foi obtida através de atividades de P\&D do "Programa de Qualificação em Grande Escala em Tecnologias Móveis - PROMOBILE", projeto patrocinado pela Samsung Eletrônica da Amazônia Ltda. nos termos da lei federal brasileira $n^{\circ} 8.248 / 91$. 


\section{Referências}

Basili, V. R., Caldiera, G. e Rombach, H. D. (1984). The Experience Factory. Em J. Marciniak (Ed.), In: Encyclopedia of Software Engineering. New York: John Wiley \& Sons.

Fassbinder, A., Delamaro, M. E. e Barbosa, E. F. (2014). Construção e Uso de MOOCs: Uma Revisão Sistemática Aracele. In: XXV Simpósio Brasileiro de Informática na Educação (SBIE 2014), 25, (pp. 332-341). Dourados.

Ferreira, L. G., Barbosa, J. L. e Gluz, J. C. (2013). Um Modelo de Recomendação Ubíqua de Conteúdo para Grupos de Aprendizes. In: XXIV Simpósio Brasileiro de Informática na Educação (SBIE 2013), 24. Campinas.

Gomes, E. H. e Pimentel, E. P. (2013). Personalização do E-Learning Baseado no Nível de Aquisição de Conhecimentos do Aprendiz. In: XXIV Simpósio Brasileiro de Informática na Educação (SBIE 2013), 24. Campinas.

Kitchenham, B. (2004). Procedures for Performing Systematic Reviews. Technical Report TR/SE 0401, Departament of Computer Science Keele University, Keele, Reino Unido.

Kitchenham, B. e Charters, S. (2007). Guidelines for performing Systematic Literature Reviews in Software Engineering. Technical Report EBSE-2007-01, School of Computer Science and Mathematics, Keele, Reino Unido.

Müller, L. e Silveira, M. S. (2013). Podes me ajudar? Apoiando a formação de pares em sistemas de ajuda em pares através de técnicas de recomendação. In: XXIV Simpósio Brasileiro de Informática na Educação (SBIE 2013), 24. Campinas.

Ninoriya, S., Chawan, P. e Meshram, B. (2011). CMS, LMS and LCMS For eLearning. In: International Journal of Computer Science Issues (IJCSI), 8, pp. 644-647.

Pontes, W. L., França, R. M., Costa, A. P. e Behar, P. (2014). Filtragens de Recomendação de Objetos de Aprendizagem: uma revisão sistemática do CBIE. In: XXV Simpósio Brasileiro de Informática na Educação (SBIE 2014), 25, (pp. 549558). Dourados.

Ramos, D. B. e Oliveira, E. H. T. (2015). Relatório Técnico da Revisão Sistemática de Literatura sobre Trilhas de Aprendizagem em Ambientes Virtuais de Aprendizagem. Universisdade Federal do Amazonas, Manaus. Fonte: http://mobmoodle.icomp.ufam.edu.br/projeto/?page_id=10

Silva, R. S. (2011). Moodle para autores e tutores. 2 ed. São Paulo: Novatec.

Tafner, E. P., Tomelin, J. F. e Müller, R. B. (2012). Trilhas de aprendizagem: uma nova concepção nos ambientes virtuais de aprendizagem - AVA. In: Congresso Internacional de Educação a Distância, 18. São Luís.

Zem-Lopes, A. M., Pedro, L. Z., Andrade, F. R., Borges, S. S. e Isotani, S. (2013). Tecnologias da Web Semântica em Ambientes Educacionais: uma Revisão Sistemática. In: XXIV Simpósio Brasileiro de Informática na Educação (SBIE 2013), 24, (pp. 977-981). Campinas. 